\title{
Advanced Fourier-based Model of Bouncing Loads
}

\author{
Vitomir Racic ${ }^{1}$, Jun Chen ${ }^{2}$, Aleksandar Pavic ${ }^{3}$ \\ ${ }^{1}$ Associate Professor \\ Department of Civil and Environmental Engineering, Politecnico di Milano \\ 32 Piazza Leonardo da Vinci, 20133 Milan, Italy \\ ${ }^{2}$ Professor \\ Department of Structural Engineering, Tongji University \\ 1239 Siping Road, 20092 Shanghai \\ People's Republic of China \\ ${ }^{3}$ Professor of Structural Dynamic \\ College of Engineering, Mathematics and Physical Sciences, University of Exeter \\ North Park Road, Exeter EX4 4QF, United Kingdom
}

\begin{abstract}
Contemporary design guideline pertinent to vibration serviceability of entertaining venues describes bouncing forces as a deterministic and periodic process presentable via Fourier series. However, fitting the Fourier harmonics to a comprehensive database of individual bouncing force records established in this study showed that such a simplification is far too radical, thus leading to a significant loss of information. Building on the conventional Fourier force model, this study makes the harmonics specific to each individual and takes into account imperfections in the bouncing process. The result is a numerical generator of stochastic bouncing force time histories which represent reliably the experimentally recorded bouncing force signals.
\end{abstract}

KEYWORDS: vibration serviceability, human-induced vibrations, human-induced excitation, stadia.

\section{INTRODUCTION}

There has been a growing number of vibration serviceability issues of floors, staircases and assembly in entertaining venues when exited by active people. One of the reasons is that crowds has become livelier than ever. In the same time substantial developments in workmanship and structural materials have reduced the mass and stiffness of a structure, yielding a natural frequency often up to $5 \mathrm{~Hz}$. This falls within the range of the body motion rates for common activities, such as bouncing and walking, yielding a large and often resonant vibration response. The final reason is a lack of adequate design guidance relevant to crowd rhythmic excitation. The IStructE/DCLG/DCMS joint Working Group design guidance on crowd dynamic loading of grandstands [1] is a step in the right direction but still not perceived as the final version. The vital refinement should address modelling the actual nature of human activities and the corresponding loads. Although there can be no absolute certainty on how a random group of people would act, the guidance is grounded on a conservative deterministic representation of crowd dynamic loading. More adequate models would portray it as a stochastic process, similar to the existing models of wind, wave or earthquake loading, all of them characterised by considerable uncertainty and randomness. The later feature this study specifically aims to address for bouncing lads. 
A reliable load model of bouncing crowds needs a reliable model of individual bouncing forces. Measured individual force time histories are characterised by considerable inter-subject variability and are invariably near-periodic [2,3], indicating their narrow band nature (Fig. 1). However, they are commonly assumed identical, perfectly periodic and presentable via Fourier series $\mathrm{F}(\mathrm{t})$ :

$$
F(t)=G \sum_{i=1}^{m} \alpha_{i} \cos \left(2 \pi i f_{b} t-\varphi_{i}\right)
$$

$\mathrm{G}$ is the body weight in the same unit as the force $\mathrm{F}(\mathrm{t})$ (typically $\mathrm{N}$ ). Coefficients $\alpha_{\mathrm{i}}$ and $\varphi_{\mathrm{i}}$ are the dominant Fourier amplitudes and phase angles corresponding to $m$ integer multiples of the bouncing rate $f_{b}$ (Fig. $1 b$ ).
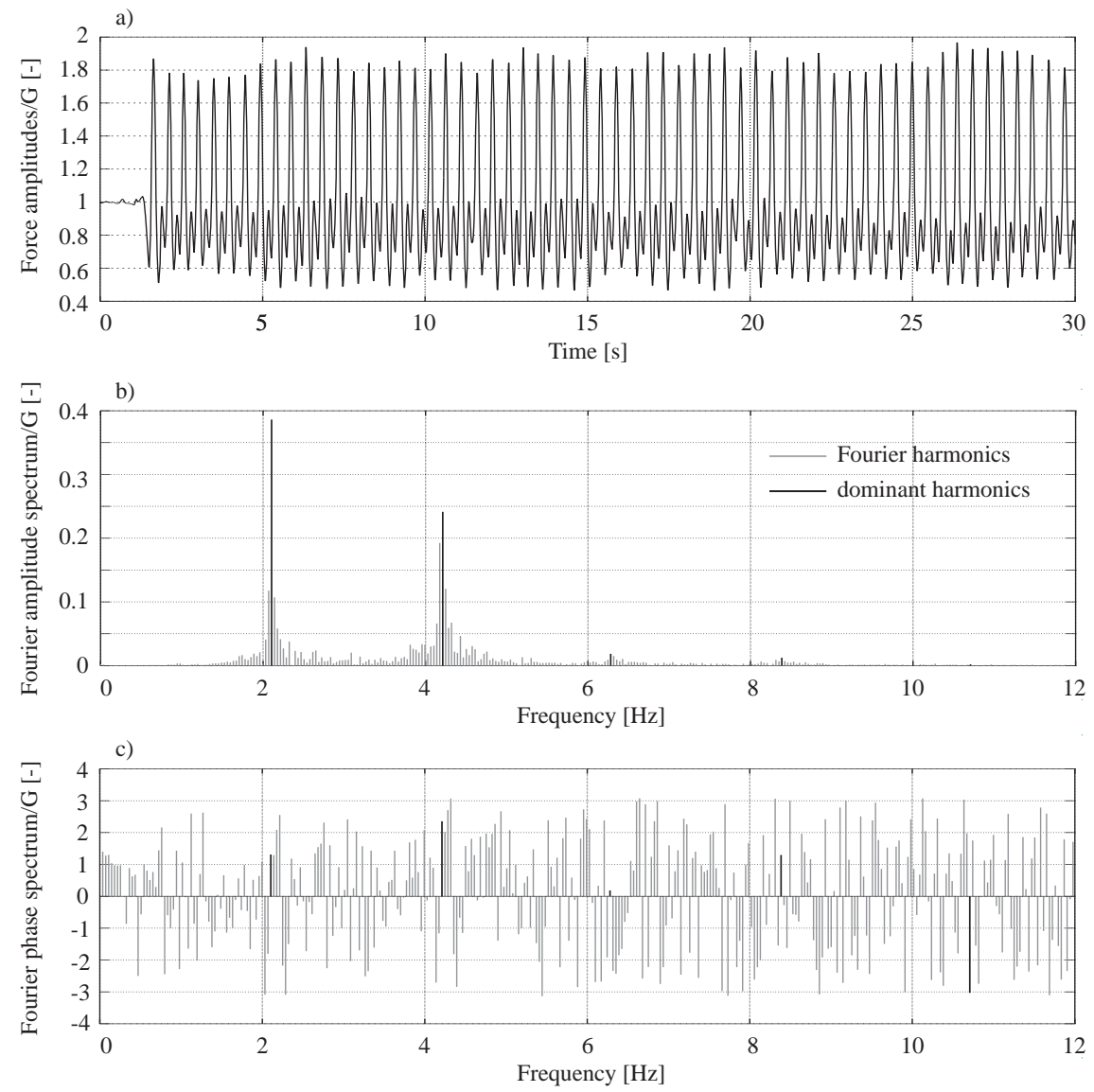

Fig.1: Example of measured bouncing (a) force-time history and its Fourier (b) amplitude and (c) phase spectra.

It is widely accepted that the modelling strategy based on Fourier harmonics leads to significant loss of information during the data reduction process [4-9]. For example, Brownjohn et al. [5] demonstrated that neglecting the energy around dominant Fourier harmonics (Fig. 1b), which is a result of uneven footfalls, yields up to $50 \%$ error in predicted vibration response. More recent study by Van Nimmen [10] showed that precision of simulated resonant vibration response primarily depends on whether variability of timing between successive footfalls is taken into account. A model of variability of successive bouncing intervals and variability of the corresponding force amplitudes are presented later in this study.

\section{EXPERIMENTAL DATA COLLECTION}

The data collection was carried out in the Light Structures Laboratory in the University of Sheffield, UK (Fig. 2).

Each participant was engaged in twelve bouncing tests, thereby generated twelve force signals. During each test a participant was asked to bounce to a steady metronome beat which was randomly selected from the frequency range $1.2-4.5 \mathrm{~Hz}$ with the 
increment of $0.3 \mathrm{~Hz}$. A test lasted between $25-45 \mathrm{~s}$, being shorter for the higher frequencies so the participant would not tire much. Rests were allowed between the tests.

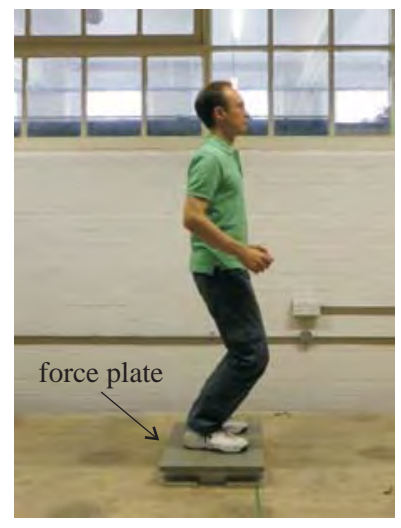

Fig. 2: Experimental setup.
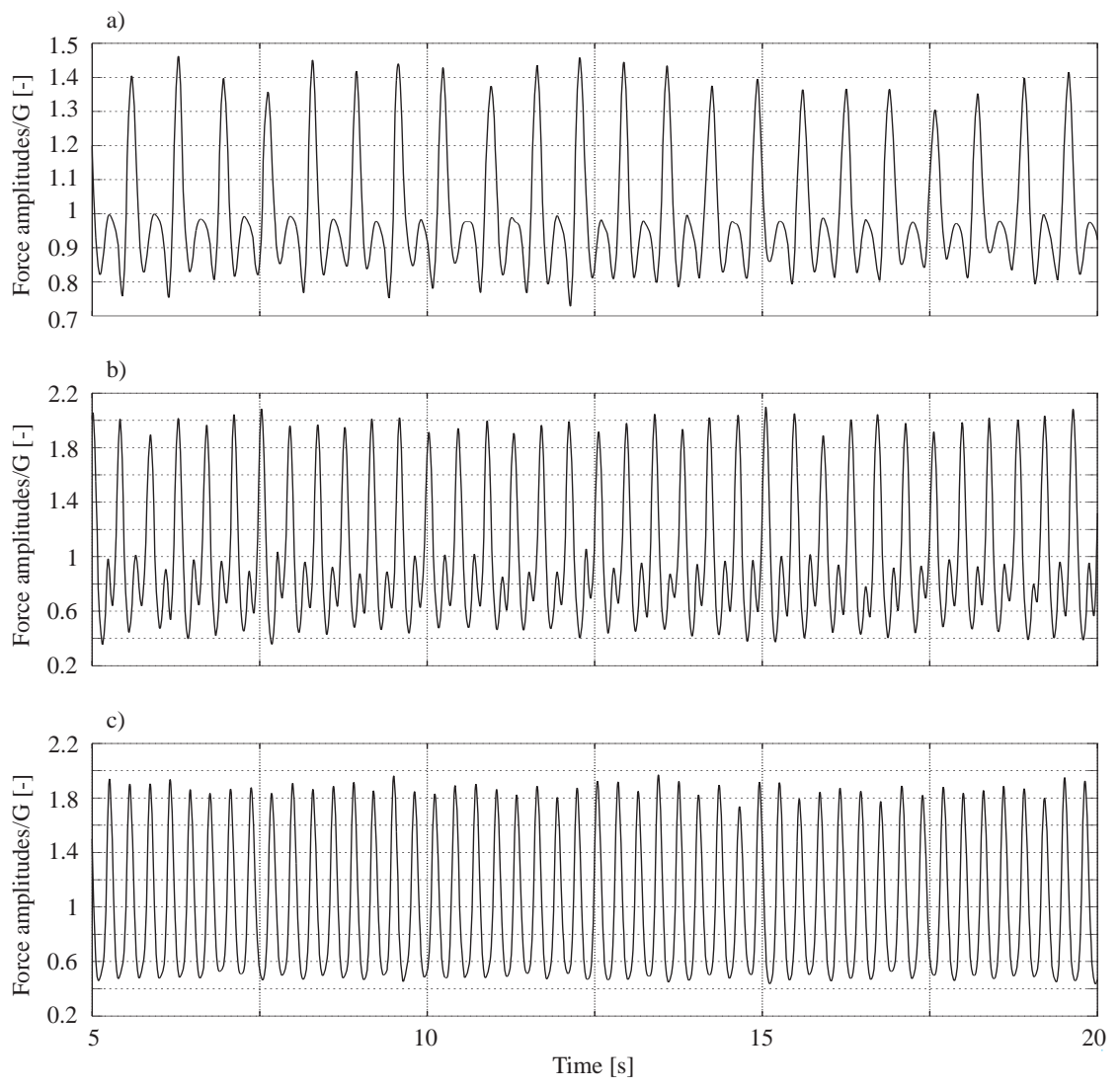

Fig. 3: Examples of bouncing force records generated at given metronome beats (a) $1.5 \mathrm{~Hz}$, (b) $2.4 \mathrm{~Hz}$ and (c) $3.3 \mathrm{~Hz}$.

The corresponding force signals were recorded by an AMTI BP-400600 force plate [11] fixed to the laboratory heavy floor (Fig. 2). In total, 80 volunteers (51 males and 29 females, body mass $72.7 \pm 15.4 \mathrm{~kg}$, height $171.2 \pm 9.2 \mathrm{~cm}$, age $26.4 \pm 7.1$ years) generated 960 vertical bouncing force signals of kind illustrated in Fig. 3. 60 signals were cast aside as some test subjects could not follow very high metronome rates. All signals were sampled at $\mathrm{fs}=200 \mathrm{~Hz}$. 


\section{MODELLING SHAPE OF FORCE SIGNALS}

This section aims to determine the "repetitive" shape of individual force signals between the successive bouncing cycles, so called "template shape". Since the vertical force amplitudes oscillate around body weight G of the test subject, the point where the amplitude is equal to the body weight and has a negative gradient was selected as starting (and completing) event of a bouncing cycle (Fig. 4).

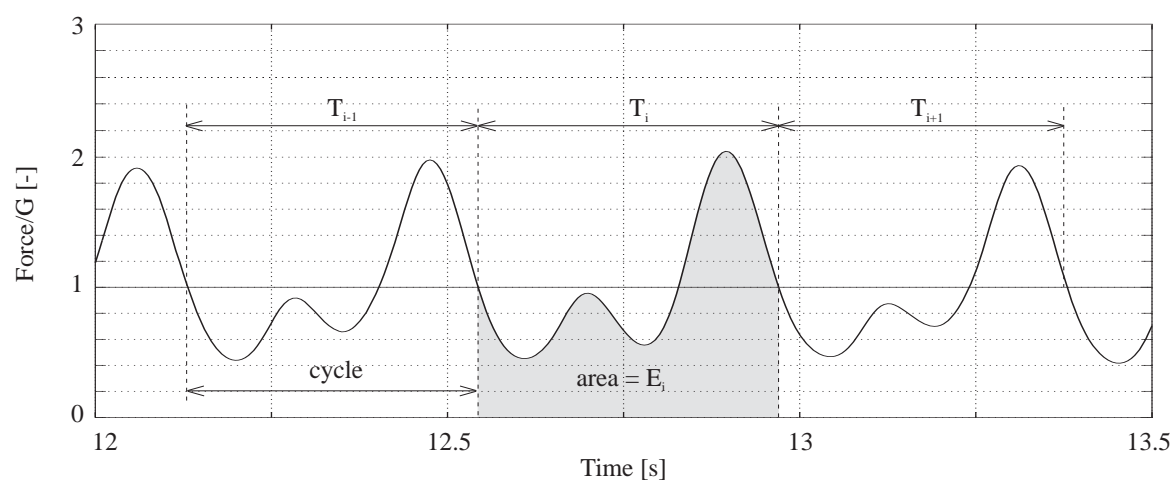

Fig. 4: A portion of the force record from Fig. 3b.

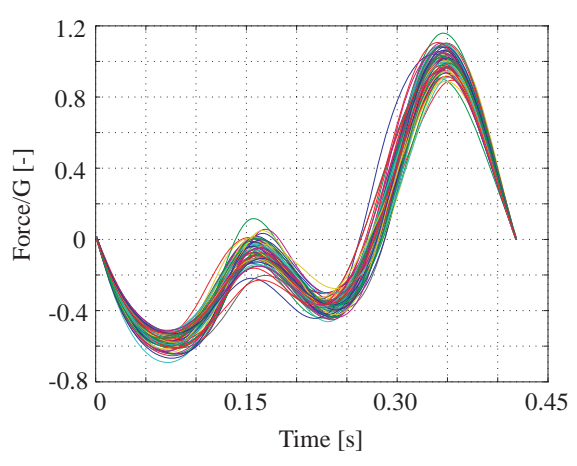

Fig. 5: Resampled G-normalised cycles for DTW.

From the $30 \mathrm{~s}$ long force signal illustrated partly in Fig. $3 \mathrm{~b}$ and 4 and yielding about 70 cycles, the central portion comprising 66 successive cycles was extracted for further analysis. The selected cycles have been normalised by the body weight G, lined up at their origins and resampled to $\mathrm{f}_{\mathrm{s}} / \mathrm{f}_{\mathrm{b}}$ data points. Fig. 5 confirms that there is a common shape that nonlinearly distorts along time and amplitude axes for successive cycles. Therefore, the template was determined as their numerical average after the common events had been aligned using dynamic time warping (DTW) method [12]. The procedure is elaborated in Racic and Chen [13] and illustrated here in Fig. 6.
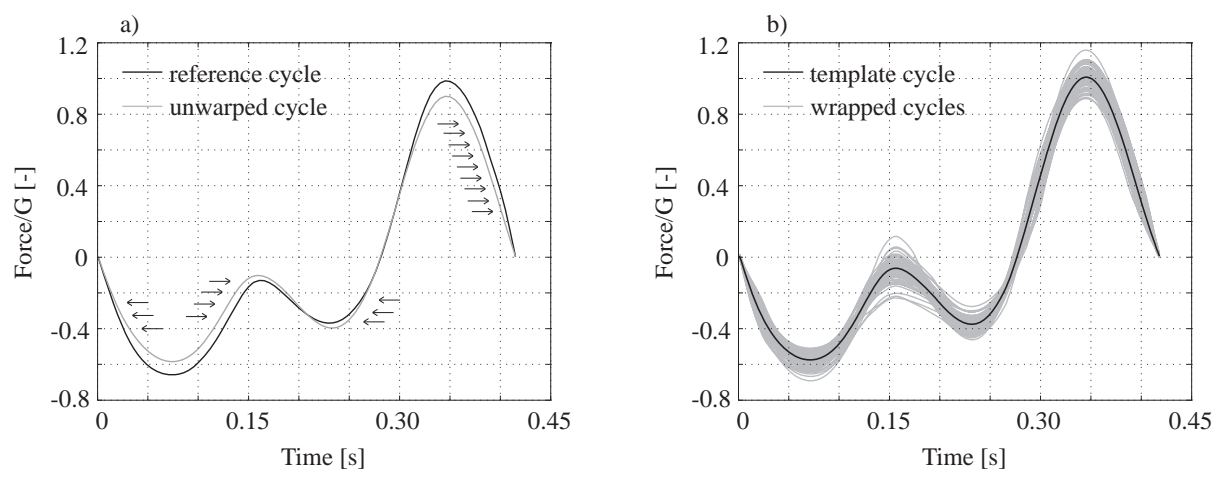

Fig. 6: (a) Illustration of DTW, and (b) template cycle. 
The shape of the template cycle $\mathrm{Z}(\mathrm{t})$ can be modelled as a sum of its first four Fourier harmonics (Equation 2). The results of the curve fitting are summarised in Fig. 7 and Table 1.

$$
Z(t)=\sum_{i=1}^{4} \alpha_{i} \cos \left(2 \pi i f_{b} t-\varphi_{i}\right)
$$

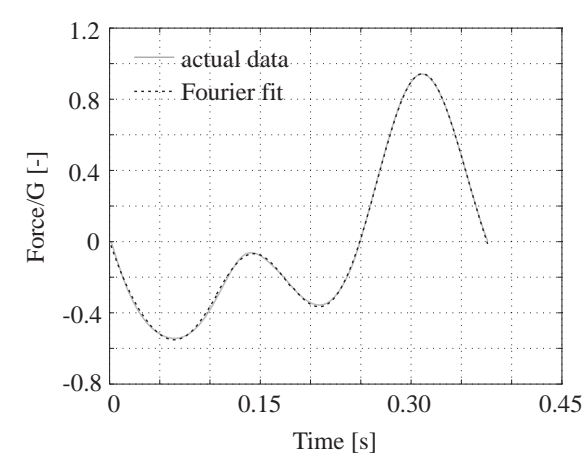

Fig. 7: Fitting the template shape.

Table 1: Results of curve fitting template shape $\mathrm{Z}(\mathrm{t})$ :

\begin{tabular}{ccccc}
\hline & \multicolumn{5}{c}{$\mathrm{f}_{\mathrm{b}}=2.40 \mathrm{~Hz}$} \\
\cline { 2 - 5 } Parameters & $\mathrm{i}=1$ & $\mathrm{i}=2$ & $\mathrm{i}=3$ & $\mathrm{i}=4$ \\
\hline$\alpha_{\mathrm{i}}[-]$ & 0.547 & 0.420 & 0.066 & 0.021 \\
$\varphi_{\mathrm{i}}[\mathrm{rad}]$ & -1.180 & -1.847 & 2.258 & 2.577 \\
\hline
\end{tabular}

\section{VARIABILITY OF CYCLE INTERVALS}

Variability of the consecutive cycle intervals $T_{i}(i=1, \ldots, 66)$ can be represented by a dimensionless series $\tau_{i}$ :

$$
\begin{aligned}
& \tau_{i}=\frac{T_{i}-\mu_{T}}{\mu_{T}} \\
& \mu_{T}=\operatorname{mean}\left(T_{i}\right)
\end{aligned}
$$

Mean of $\tau_{i}$ is zero and its auto spectral density (ASD) can be calculated as [14]:

$$
S_{\tau}\left(f_{m}\right)=\frac{A_{\tau}^{2}\left(f_{m}\right)}{2 \Delta f}, f_{m}=\frac{m}{66}, \quad m=0, \ldots, 32
$$

where $A_{\tau}\left(f_{m}\right)$ is a single-sided discrete Fourier amplitude spectra and $\Delta f=1 / 66$ is the spectral line spacing (Fig. 8).

The variance of $\tau_{i}$ is the integral of the ASDs (Newland 1993). Unlike random number generators, such as probability density functions, the ASD contains information of the frequency content of $\tau_{i}$ series. Hence, assuming that a test subject maintains the same bouncing style for any period of bouncing, a model of the ASD can be used to synthesise artificial $\tau_{k}^{\prime} \quad(k=$ $1, \ldots, N)$ series of arbitrary length (e.g. $N \gg 66$ ) with the statistical properties of the actual $\tau_{i}$ series.

The $\operatorname{ASD} S_{\tau}\left(f_{m}\right)$ can be analytically described by a sum of 33 equidistant Gaussians (Fig. 8):

$$
S_{\tau}^{\prime}(f)=\sum_{j=1}^{33} W_{j} e^{-\frac{\left(f-c_{j}\right)^{2}}{2 b^{2}}}
$$


If the Gaussian centres $c_{j}$ are placed in each data point on the quasi-frequency axis and all Gaussian bells have the same predefined widths $b=\Delta f$, their heights $W_{j}$ can be optimised using the non-linear least-square method [15] to fit exactly the actual ASD (Fig. 8).

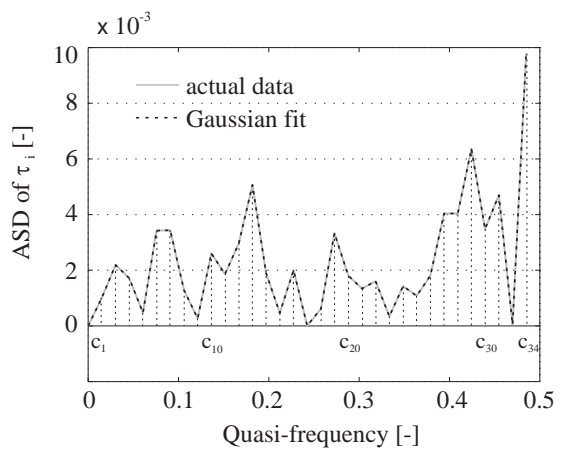

Fig. 8: ASD of $\tau_{i}$ series and its curve fit.

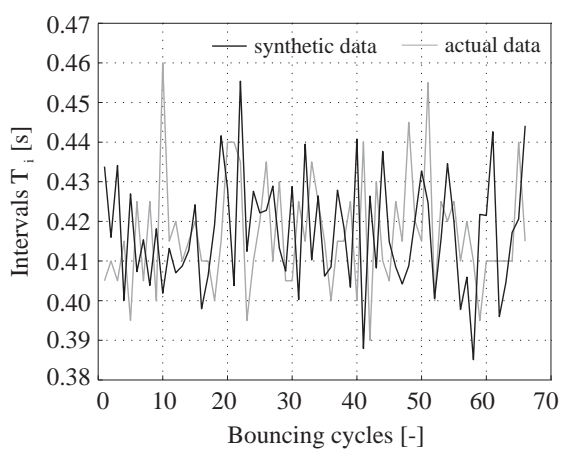

Fig. 9: Measured and an example of synthetic cycle intervals.

To create a series of synthetic cycle intervals $T_{k}^{\prime}(k=1, \ldots, N)$, Equation (5) first calculates $S_{\tau}^{\prime}\left(f_{n}\right)$ values at equidistant data points $f_{n}=n \Delta f^{\prime}$, where $n=0, \ldots, N / 2-1$ and $\Delta f^{\prime}=1 / N$. These are then used in Equation (4) to compute the corresponding Fourier amplitudes $A_{\tau}^{\prime}\left(f_{n}\right)=\sqrt{2 \Delta f^{\prime} S_{\tau}^{\prime}\left(f_{n}\right)}$. Assuming the uniform distribution of the phase angles in the range $[-\pi, \pi], A_{\tau}^{\prime}\left(f_{n}\right)$ are then fed into the inverse FFT algorithm to generate $\tau_{k}^{\prime}$ series. Different $\tau_{k}^{\prime}$ series with the same variance and the frequency content can be created by varying the sets of random phase angles. Finally, multiplying $\tau_{k}^{\prime}$ by $\mu_{T}$ and adding the offset $\mu_{T}$, yields a synthetic $T_{k}^{\prime}$ series (Fig. 9). Working under assumption that a test subject keeps the same bouncing style in a narrow range of bouncing rates, $\mu_{T}$ can take a slightly different value $\mu_{T}{ }^{\prime}$ to generate cycle intervals corresponding to rate $1 / \mu_{T}^{\prime}$.

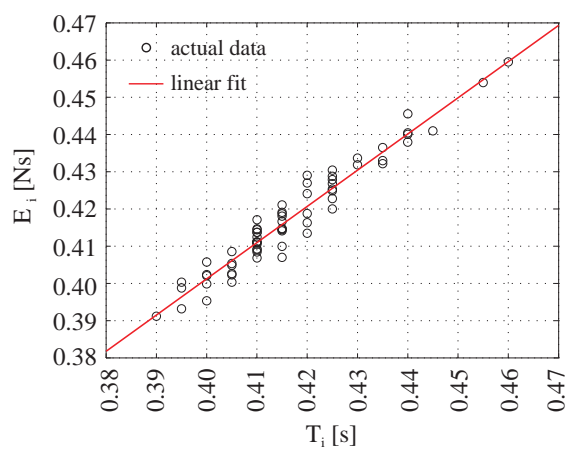

Fig. 10: Cycle energy $E_{i}$ vs. cycle intervals $T_{i}$.

\section{VARIABILITY OF FORCE AMPLITUDES}

Energy of bouncing cycles $E_{i}$ can be defined as the integral of the weight -normalised force amplitudes over the corresponding cycle intervals $T_{i}$ (Fig. 4).

The relationship between the two parameters (Fig. 10) can be described by the following linear regression model:

$$
E_{i}=\rho_{1} T_{i}+\rho_{0}+\Delta E_{i}
$$

Parameters $\rho_{1}=0.972$ and $\rho_{0}=0.012$ are regression coefficients and $\Delta E_{i}$ is a disturbance term, typically modelled as a random Gaussian noise [16].

Hence, having generated synthetic cycle intervals $T_{k}^{\prime}(k=1, \ldots, N)$, the corresponding energies $E_{k}^{\prime}$ can be calculated using Equation (6). The energies are then assigned to a sequence of $N$ bouncing cycles by scaling the amplitudes of the template shape with factors $\xi_{k}$ : 


$$
\xi_{k}=\frac{E_{k}^{\prime}}{E_{t c}}
$$

where $E_{t c}$ is the energy of the template shape. The empirical evidence is provided in the next section.

\section{ARTIFICIAL FORCE SIGNALS}

Fig. 11 and 12 illustrate an example of synthetic force signal. Comparison of Fig. 11a to 11b and Fig. 12a to 12b highlights the close match with the actual force record in both time and frequency domain.
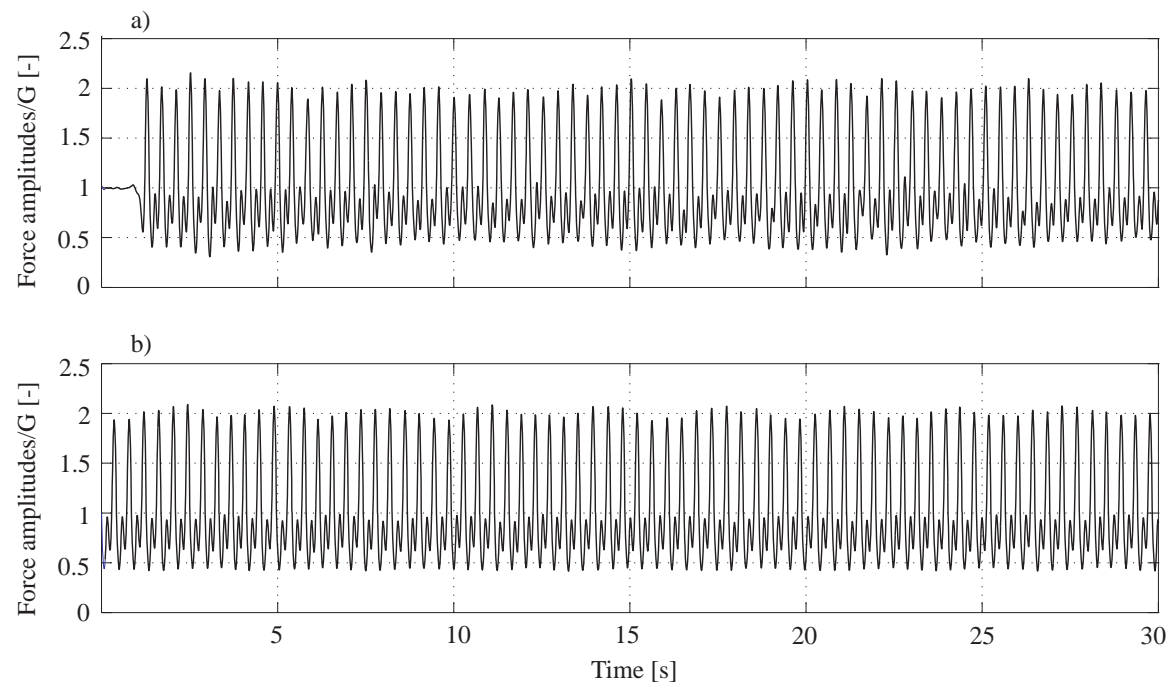

Fig. 11: (a) Measured and (b) an example of synthetic force-time hisory.
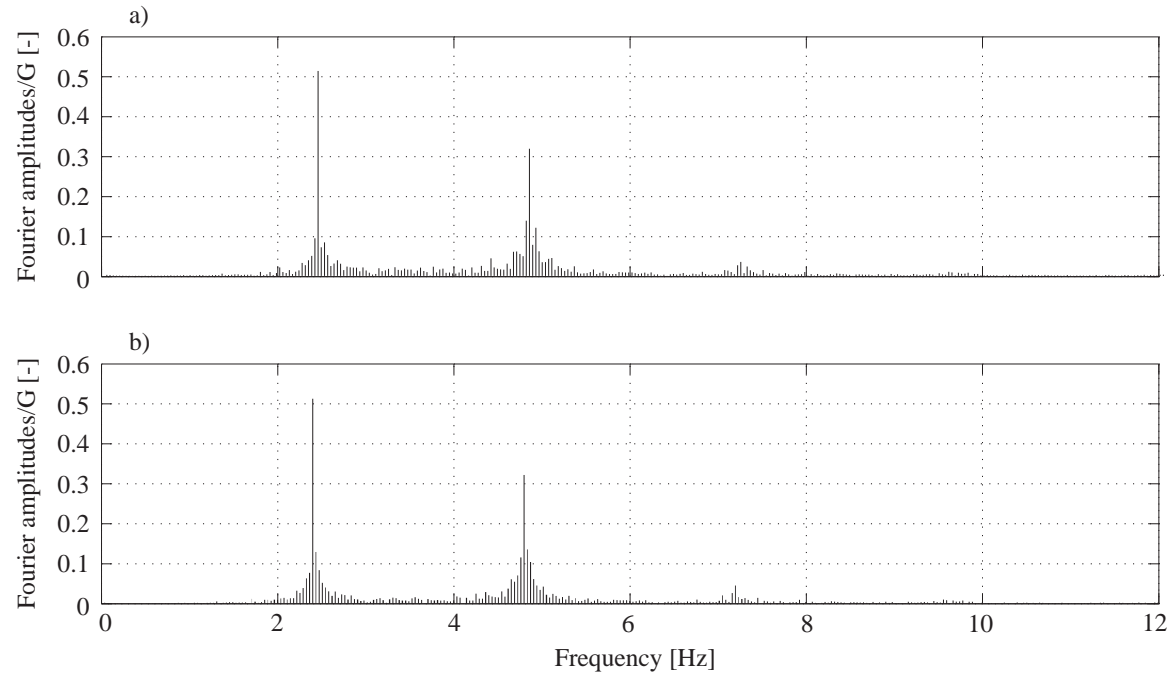

Fig. 12: Discrete Fourier amplitude spectra of the time series shown in Fig. 11.

\section{GENERATOR OF RANDOM FORCE SIGNALS}

Parameters of the template shape $Z(t)$, the ASD $S_{\tau}^{\prime}(f)$, disturbance term $\Delta E_{i}(t)$ and the regression coefficients $\rho_{1}$ and $\rho_{0}$, were extracted from each of the 900 force signals recorded in Section 2 and stored in in 900 MATLAB structure files [17], here called "mat files". The mat files were classified into $0.3 \mathrm{~Hz}$ wide clusters which centres correspond to the 12 bouncing rates in the range 1.2-4.5 $\mathrm{Hz}$ given during the data collection. It can be assumed that the modelling parameters stored in any 
mat file within a cluster can be used to generate synthetic force signals at any bouncing rate within the cluster's frequency range.

The flow chart in Fig. 13 presents the algorithm to generate an artificial force signal. A more elaborate explanation behind the process as well as the examples of synthetic forces can be found in Racic and Chen [13].

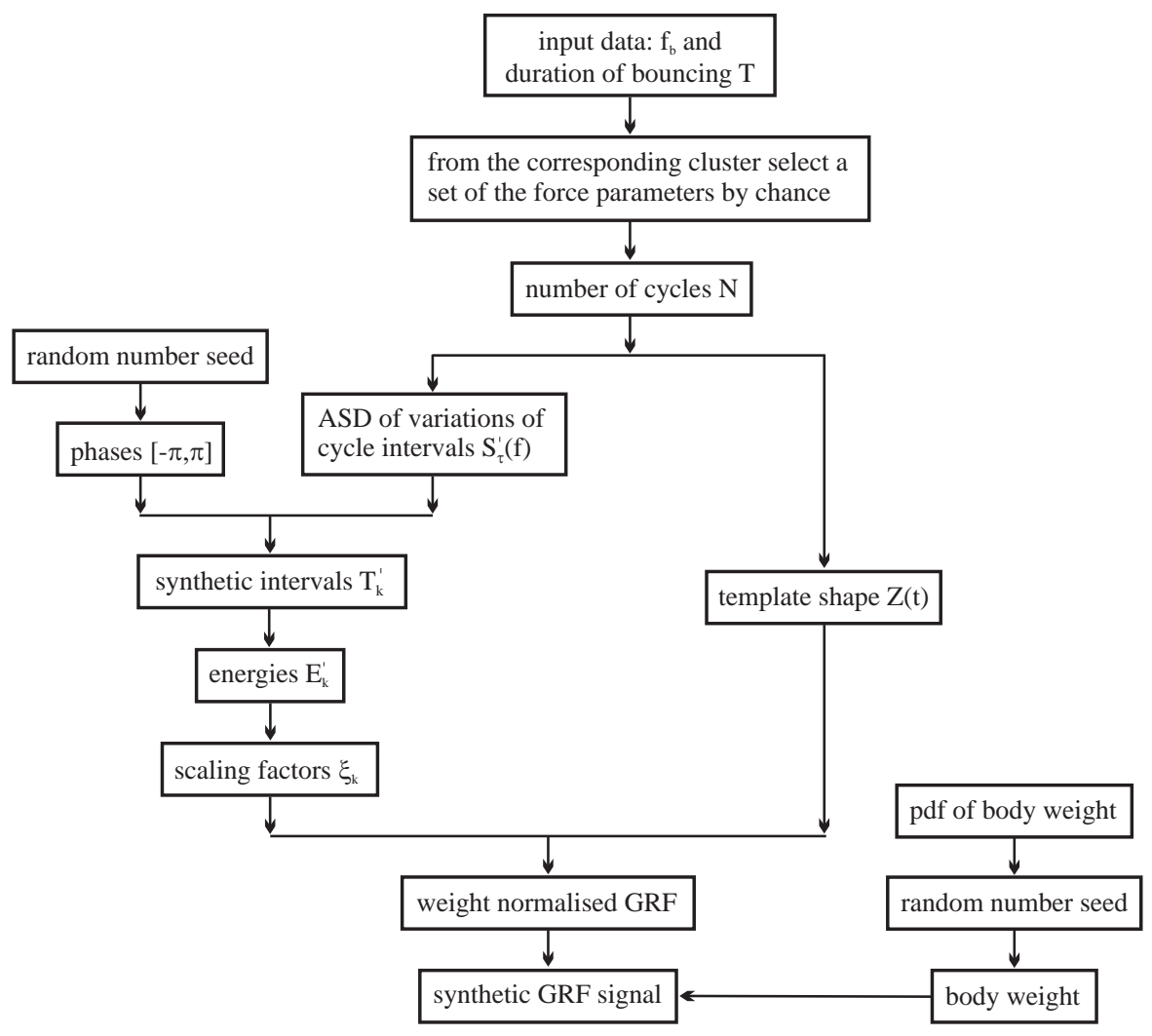

Fig. 13: Flow chart.

\section{SUMMARY AND CONSCLUSIONS}

This study combines the traditional Fourier modelling approach with novel models of variability of timing and amplitudes/energy of the successive bouncing cycles, yielding a numerical generator of random near-periodic bouncing force time histories that look like measured. The modelling parameters are extracted from a large database of bouncing force records, classified and stored in narrow clusters with respect to the bouncing rate. Assuming that the forces generated at very close rates have similar shape, amplitudes and level of variability, modelling parameters within a cluster can be used to generate synthetic forces at any rate within the cluster's frequency range. The key modelling parameters are random variables, so two identical forces can be generated only by chance.

However, there is still a room for improvement. The current version of the model runs on the modelling parameters extracted from the force records measured on a rigid laboratory floor. Hence, it can be used to study only cases of incipient dynamic instability, i.e. when the vibration level is not too much perceptible to the occupant and therefore does not affect his/her bouncing motion. An elaborate database of bouncing force records generated by many individuals bouncing at different rates on more or less vibrating surfaces still waits to be established. So is the case with bouncing loads generated by groups of different sizes, with or without the motion of the structure itself. There are many more factors affecting human-induced loads, such as different auditory, visual and tactile stimuli. Moreover, there is still a gap in the knowledge on the interaction between individuals in groups and crowds and its influence on the corresponding net dynamic loads on the structure, which still need to be studied, measured and modelled. 


\section{ACKNOWLEDGEMENTS}

The authors would like to acknowledge the financial support provided by PRIN 2015-2018 "Identification and monitoring of complex structural systems" and National Natural Science Foundation of China 347 (51478346) and State Key Laboratory for Disaster Reduction of Civil Engineering (SLDRCE14-B-16). Also, the author would like to thank all test subjects for participating in the data collection.

\section{REFERENCES}

[1] IStructE/DCLG/DCMS Working Group (2008) Dynamic performance requirements for permanent grandstands subject to crowd action: Recommendations for management, design and assessment. The Institution of Structural Engineers, The Department for Communities and Local Government and The Department for Culture Media and Sport, London, UK..

[2] Sim JHH, Blakeborough A, Williams M (2008) Statistical model of crowd jumping loads. ASCE Journal of Structural Engineering 134 (12), 1852-1861.

[3] Parkhouse JG, Ewins DJ (2006) Crowd-induced rhythmic loading. Structures \& Buildings 159 (SB5), 247-259.

[4] Rainer J. H., Pernica G., Allen D.E. (1988) Dynamic loading and response of footbridges. Canadian Journal of Civil Engineering 15 (1), 66-71.

[5] Brownjohn JMW, Pavic A, Omenzetter P (2004) A spectral density approach for modelling continuous vertical forces on pedestrian structures due to walking. Canadian Journal of Civil Engineering 31, 65-77.

[6] Zivanovic S, Pavic A, Reynolds P (2005) Human-structure dynamic interaction in footbridges. Bridge Engineering 158 (BE4), 165-177.

[7] Racic V, Pavic A (2010) Stochastic approach to modelling near-periodic jumping force signals. Mechanical Systems and Signal Processing 24, 3037-3059.

[8] Racic V, Brownjohn JMW (2012) Mathematical modelling of random narrow band lateral excitation of footbridges due to pedestrians walking. Computers \& Structures 90-91, 116-130.

[9] Racic V, Morin JB (2014) Data-driven modelling of dynamic excitation of bridges induced by people running. Mechanical Systems and Signal Processing 43, 153-170.

[10] Van Nimmen K, Lombaert G, Jonkers I, De Roeck G, Van den Broeck P (2014) Characterisation of waking loads by 3D inertial motion tracking. Journal of Sound and Vibration 33 (20) 5212-5226.

[11] AMTI (2014) AMTI user manuals www.amti.biz

[12] Holmes JR, Holmes W (2001) Speech synthesis and recognition, $2^{\text {nd }}$ edn. Taylor and Francis, London, UK.

[13] Racic V, Chen J (2015) Data-driven generator of stochastic dynamic loading due to people bouncing. Computers and Structures 158, 240-250.

[14] Newland DE (1993) An introduction to random vibrations, spectral and wavelet analysis, $3^{\text {rd }}$ edn. Pearson Education Limited, Harlow, UK.

[15] Bates DM, Watts DG (1998) Nonlinear regression and its applications. Wiley, New York, USA.

[16] Bishop CM (2006) Pattern recognition and machine learning, $4^{\text {th }}$ edn. Springer, New York, USA.

[17] MathWorks (2014) Matlab user guides www.mathworks.com 